\title{
Carbon nanotube isolation layer enhancing in-liquid quality-factors of thin film bulk acoustic wave resonators for gravimetric sensing
}

\author{
Girish Rughoobur ${ }^{\mathrm{a}, 1}$, Hisashi Sugime ${ }^{\mathrm{a}, 2}$, Mario DeMiguel-Ramos ${ }^{\mathrm{a}}$, Teona \\ Mirea $^{\mathrm{b}}$, Shan Zheng ${ }^{\mathrm{a}}$, John Robertson ${ }^{\mathrm{a}}$, Enrique Iborra ${ }^{\mathrm{b}}$, Andrew John \\ Flewitt ${ }^{\mathrm{a}, *}$
${ }^{a}$ Electrical Engineering Division, Department of Engineering, University of Cambridge, 9 JJ Thomson Avenue, Cambridge, CB3 OFA, UK
${ }^{b}$ GMME-CEMDATIC-ETSI de Telecomunicación, Universidad Politécnica de Madrid, 28040 Madrid, Spain

\begin{abstract}
A thickness longitudinal mode (TLM) thin film bulk acoustic resonator biosensor is demonstrated to operate in water with a high quality-factor, $Q$. This is achieved using a layer of carbon nanotubes (CNTs) on top of the resonator which has a significantly different acoustic impedance to either the resonator or liquid whilst being susceptible to the binding of biological molecules. This allows the resonance to be decoupled from direct energy loss into the liquid, although still retaining mass sensitivity. AlN solidly mounted resonators (SMRs) having a thickness shear mode (TSM) at $1.1 \mathrm{GHz}$ and TLM at $1.9 \mathrm{GHz}$ are fabricated. CNTs with different forest densities are grown by chemical vapor deposition on the active area with Fe as catalyst and resulting devices compared. High forest density CNTs are shown to acoustically decouple the SMRs from the water and in-liquid TLM $Q$ values higher than 150 are recorded even exceeding TSM SMRs without CNTs. The TLM $Q$ in water is remarkably improved from 3 to 160

\footnotetext{
${ }^{*}$ Corresponding author at Electrical Engineering Division, Department of Engineering, University of Cambridge, 9 JJ Thomson Avenue, Cambridge, CB3 0FA, UK, Phone: +44 (0) 1223 748332. Fax: +44 (0) 1223748332

Email address: ajf@eng.cam.ac.uk (Andrew John Flewitt)

${ }^{1}$ Present address: Microsystems Technology Laboratories, Massachusetts Institute of Technology, 60 Vassar Street, Cambridge, MA 02139, USA

${ }^{2}$ Present address: Waseda Institute for Advanced Study, Waseda University, 1-6-1 Nishi Waseda, Shinjuku-ku, Tokyo 169-8050, Japan
} 
for the first time by dense CNT forests, rendering the large-scale fabrication of TLM SMRs for liquid-phase sensing applications possible. Despite this partial isolation, SMRs with CNT forests $\sim 15 \mu \mathrm{m}$ tall can still detect binding of bovine serum albumin.

Keywords: Carbon nanotubes, Bulk wave resonators, Gravimetric sensing, Acoustic wave decoupling, In-liquid sensors

\section{Introduction}

In recent years the need for minature, low cost, label-free and ultra-sensitive chemical and biological sensors has grown significantly. Gravimetric sensors based on acoustic wave resonators, which have been predominantly used in 5 r.f. filters, are ideal to achieve these objectives. Mass accretion on the sensor surface (which can be functionalized to only permit specific molecules to attach) decreases the resonant frequency, $f_{\mathrm{r}}$; this is the basis for sensing. This frequency shift, $\Delta f_{\mathrm{r}}$, and hence the mass sensitivity, $S_{\mathrm{m}}$, are proportional to $f_{\mathrm{r}}^{2}$ according to the Sauerbrey equation [1]. Better mass sensitivities are there-

10 fore achievable by operating at higher frequencies. Acoustic wave resonators can be broadly categorised into surface acoustic wave (SAW) and bulk acoustic wave (BAW) resonators. SAWs are generated on a piezoelectric material by applying an electrical signal to a set of interleaved electrodes known as interdigitated transducers (IDTs), which are a comb-like array [2]. Typically SAWs

15 are guided along the free surface and decay exponentially with depth $(>90 \%$ within one wavelength from surface) while BAWs propagate freely like in an infinite medium [3]. Higher $f_{\mathrm{r}}$ through miniaturisation of IDTs in SAW resonators is difficult to achieve due to lithography constraints [4]. In addition, at higher $f_{\mathrm{r}}$, SAW resonators have lower $Q$-factors and limited power handling capabilities [5], although the mass sensitivity has been shown to improve when increasing the operating frequency from $80 \mathrm{MHz}$ to $1 \mathrm{GHz}$ [6]. Quartz crystal microbalances (QCMs) are the most well-established BAW gravimetric sensors; these use quartz plates (330-55 $\mu \mathrm{m}$ thick) and operate generally in a shear mode 
with frequency $(5-20 \mathrm{MHz})$ [7, 8]. To increase the $f_{\mathrm{r}}$, expensive processes are necessary to thin the quartz plates, which eventually become fragile. However higher frequency $(1-5 \mathrm{GHz})$ resonators have been amenable by thin film technology, through the emergence of thin film BAW resonators.

Thin film BAW resonators generally consist of a thin piezoelectric film (0.5-3 $\mu \mathrm{m}$ thick) of aluminium nitride ( $\mathrm{AlN})$ or zinc oxide $(\mathrm{ZnO})$ "sandwiched" between two metallic electrodes [9]. The application of an a.c. signal across the electrodes generates a standing wave confined in the piezoelectric layer, which resonates at a frequency that is determined by the film thickness [10]. Depending on the method of isolating the acoustic wave from the substrate, two types of BAW resonators can be distinguished: film bulk acoustic resonators (FBARs) and 35 solidly mounted resonators (SMRs). FBARs use air for acoustic isolation; SMRs have an acoustic reflector consisting of layers alternating between low acoustic impedance and high acoustic impedance [9]. Thin film BAW resonators are normally operated in the thickness longitudinal mode (TLM), which is excited in piezoelectric films with a $c$-axis oriented parallel to the resonator surface normal.

${ }_{40}$ Thus the application of an r.f. signal causes the mechanical deformation to be parallel to the direction of wave propagation [11]. Biosensing with longitudinal mode thin film BAW resonators is usually performed in dry conditions as the TLM couples efficiently into liquids leading to severe damping ( $>90 \%$ drop in quality-factors at resonance, $Q$ ) [12]. This prevents TLM resonators from being used in liquid environments, which is a major hindrance for both chemical and biological sensing as it requires surfaces to be dry when measurement takes place. Thus far the thickness shear mode (TSM) resonance has been adopted for biosensing applications because it does not compress in liquids with low viscosity and hence has lower attenuation [13].

In-liquid TSM resonators in the $\mathrm{GHz}$ regime have already been shown in the real-time measurement of antibody-antigen binding [14], study of DNA hybridization [15, 16] and organophosphorus pesticide detection [17]. TSM resonances can be excited effectively by using a piezoelectric layer with a $c$-axis inclined between $20^{\circ}-40^{\circ}$ with respect to the surface normal, and this is achieved 
55 by an off-axis growth of the film as reported in [18, 19, 20, 21]. Both the TSM and the TLM are stimulated (quasi-shear and quasi-longitudinal modes) in such piezoelectric films, with the TSM resonating at a lower frequency compared with the TLM because of the lower propagating velocity of the shear wave. Due to this lower $f_{\mathrm{r}}$, the mass sensitivity of the TSM is only around one-third of the 60 corresponding parameter for TLM resonators having similar dimensions [14]. In addition, damping still occurs when the shear resonance propagates through more viscous liquids [12, 22]. Crucially, the scalability of fabricating inclined $c$-axis films is also limited compared to highly oriented $c$-axis films due to significant and expensive modifications necessary for the depositions as reported ${ }_{65}$ in previous works [23, 24]. Alternative methods using either seed layers such as AlN [19, 12] with specific crystallographic orientations or Al electrodes with controlled roughness [25] improve the uniformity of inclined $c$-axis piezoelectric films over a short range but still cannot achieve homogeneity over larger substrates. It would therefore be preferable to find a means of using the TLM for

70 in-liquid sensing. Attempts to confine the liquid using microchannels have been attempted to reduce damping in the TLM [26, 27]. However the attenuation of the TLM is very sensitive to the channel thickness, which needs to be precise to prevent large energy loss into the liquid [26]. The second harmonic of the TLM has also been reported for in-liquid sensing, although at higher $f_{\mathrm{r}}$, low $Q$

75 of 40 are achieved, which reduces the measurement accuracy of $\Delta f_{\mathrm{r}}$ and hence $S_{\mathrm{m}}$ [28]. Pottigari and Kwon [29] designed FBARs with a vacuum gap to isolate the resonator from liquid interaction by using a parylene membrane held by microposts. Even though the measured in-liquid $Q$ was $\sim 140$, the area of the resonator in contact with vacuum forms a parallel resonator with the area under

so the posts, which significantly reduces the sensitivity to mass variations on the membrane. The fragility of such a device also limits its potential for in-liquid TLM thin film BAW sensors.

In this work, a new approach using an interface layer between the resonator and the liquid to enable TLM devices for working in-liquid is presented. Rather ${ }_{85}$ than isolating the resonator entirely from the liquid, the purpose of this layer 
is to partially isolate the TLM and TSM from the liquid by engineering the thickness of this interface layer. In addition the interface layer itself can be functionalized to allow mass attachment (from molecules in the liquid), which is detected by the resonant acoustic wave. In this way, the interface layer decouples

90 the resonant device from the process of mass attachment whilst retaining mass sensitivity in the liquid. This is demonstrated using a layer of carbon nanotubes (CNTs), which is grown directly on the active area of the resonator.

Owing to their extraordinary electronic and mechanical properties (stiffness coefficient $\sim 1 \mathrm{TPa}$, acoustic velocity $\sim 62 \mathrm{~km} \cdot \mathrm{s}^{-1}$ ) 30, 31], CNTs have tremendous potential for application in gravimetric biosensors. In addition to their compatibility and affinity to biological compounds [32], the larger surface area to volume ratio of CNTs makes them an attractive alternative to metallic electrodes for improving the sensitivities and detection limits of thin film BAW biosensors. Indeed CNT integration on the surface increases the binding area for molecules without physically increasing the dimensions of the sensor. Consequently, for the same concentration of a biological sample, a larger number of targeted molecules can be bound onto the resonator surface compared to devices with metal electrodes; both $\mathrm{ZnO}$ [33] and AlN 34] thin film BAW resonators with higher sensitivities attributed to the presence of a CNT layer have already been demonstrated in air using the TLM.

When the CNTs are grown directly on substrates, they form different morphologies depending on their number density. With number densities as high as $\sim 10^{11}-10^{13} \mathrm{~cm}^{-2}$, the growth of vertically-aligned (VA) CNT forests is favored 35]. Although in this work only the forest number density will be considered, the low mass densities of CNT forests ranging from $0.03 \mathrm{~g} \cdot \mathrm{cm}^{-3}$ (Futaba et al. [36]) to $1.6 \mathrm{~g} \cdot \mathrm{cm}^{-3}$ (Sugime et al. [37]) effectively reduce mass loading on the resonators compared to metal electrodes. Depending on their morphology, CNTs can have different acoustic impedances, which can be tuned to ensure a sufficient mismatch with the acoustic impedance of the piezoelectric 115 material to confine the acoustic wave in the resonator [31, 38]. CNTs have a further merit compared to metals as they can be functionalized for direct cova- 
lent bonding to molecules, or for non-covalent bondings by means of different types of polymers 39] thus preventing non-specific binding or the need for additional binding layers. Moreover the chemical bonding structure of CNTs, which consists of carbon atoms only, is attractive for detecting organic compounds. Hence, the potential exists to use functionalized CNT layers, which would function both as electrodes and as sensing layers [40]; however electrodes made only with CNTs introduce parasitic series resistances, which deteriorate the $Q$-factor values at resonance in air according to [41].

To assess the viability of CNTs functioning as an interface layer, inclined $c$-axis AlN based SMRs operating at fundamental TSM and TLM frequencies of $\sim 1 \mathrm{GHz}$ and of $\sim 2 \mathrm{GHz}$ respectively are fabricated. The CNTs are grown by chemical vapor deposition (CVD) on the active area so that they act solely as an acoustic decoupling layer to mitigate acoustic energy loss in a liquid environment. The impact of this CNT layer on the TSM and TLM resonances are investigated and compared to resonators without a CNT layer. Three different CNT forest densities are investigated ranging from densely-packed CNT forests to "spaghetti"-like tubes. Additionally the mass sensitivities in liquid of CNT coated SMRs are also evaluated.

\section{Material and methods}

The SMRs shown schematically in Figure 1 (a) are fabricated on (100)oriented, $n$-type, $500 \mu \mathrm{m}$ thick $\mathrm{Si}$ wafers with diameter $100 \mathrm{~mm}$, which have been thermally oxidized (wet-oxidation) to obtain $\sim 620 \mathrm{~nm}$ of $\mathrm{SiO}_{2}$. Four reflector layers consisiting of alternating $\mathrm{Mo}(\sim 650 \mathrm{~nm})$ and $\mathrm{SiO}_{2}(\sim 620 \mathrm{~nm})$ are

140 then sputtered sequentially in a Leybold Z500 sputtering system to achieve a reflection centered around a frequency of $2.2 \mathrm{GHz}$ for the longitudinal mode. The top reflector layer is polished mechanically using alumina slurry to reduce the roughness to less than $2 \mathrm{~nm}$. $\operatorname{Ti} / \operatorname{Ir}(\sim 20 / 130 \mathrm{~nm})$ is deposited as a bottom electrode by electron beam evaporation and $400 \mathrm{~nm}$ of Mo is deposited by sputtering as a hard mask. With standard UV photolithography this hard 
mask of Mo is patterned and etched by commercial aluminium etchant. The exposed Ir is then etched by Ar ion-milling followed by dry etching of Ti with $\mathrm{SF}_{6}$ chemistry to define the bottom electrode. Subsequently the Mo hard mask is removed by commercial $\mathrm{Al}$ etchant. AlN sputtering is performed in an in-house ultra-high-vacuum system, pumped to a base pressure below $8 \times 10^{-7} \mathrm{~Pa}$. A pulsed DC source (MKS ENI 235, Andover, MA, USA) operating at $250 \mathrm{kHz}$ powers a high purity (99.999\%), $150 \mathrm{~mm}$ diameter Al target. An AlN seed layer ( $\sim 100 \mathrm{~nm}$ thick) is grown prior to the piezoelectric AlN, at high pressure (0.66 $\mathrm{Pa}$ ) and $600 \mathrm{~W}$ without intentionally heating or biasing the substrate. With these conditions seed layers with mainly (103) orientation are deposited, which are essential to promote the growth of the subsequent AlN films with inclined grains as shown in Figure 1 (c) 19]. AlN films with the wurtzite $c$-axis inclined up to $24^{\circ}$ (Figure 1 (c)) with respect to the surface normal in the substrates placed between 2 and $5 \mathrm{~cm}$ from the target axis. The piezoelectric AlN films 160 ( 1-1.2 $\mu \mathrm{m}$ thick) are sputtered at lower pressure $\left(0.27 \mathrm{~Pa}\right.$, in a $60 \% \mathrm{~N}_{2}$ in $\mathrm{Ar}$ admixture), maintaining the substrate at $550{ }^{\circ} \mathrm{C}$ and applying r.f. bias of $-50 \mathrm{~V}$ to keep the residual stress below $200 \mathrm{MPa}$. The top electrode of Mo ( 150 nm) is sputtered in a DC magnetron sputtering system (Metallifier Sputter Coater, Precision Atomics, Cambridge, UK) from a 99.95\% Mo target at a power of 100 $165 \mathrm{~W}$ in a $0.35 \mathrm{~Pa} \mathrm{Ar}$ atmosphere. This top electrode is then patterned by standard UV photolithography and dry etched with reactive ion etching using $\mathrm{CF}_{4} / \mathrm{O}_{2}$ chemistry. For the devices with CNTs, the active area (a pentagon inscribed in an $80 \mu \mathrm{m}$ diameter circle, Figure 1 (b) forming an active area of $1.42 \times 10^{-4}$ $\left.\mathrm{cm}^{2}\right)$ is defined using standard UV photolithography. $\mathrm{Al}$ (8 $\mathrm{nm}$ thick) and Fe (thickness ranging from $0.5 \mathrm{~nm}$ to $4.5 \mathrm{~nm}$ ) are deposited in a thermal evaporator (E306, Edwards, West Sussex, UK) at a base pressure of $2 \times 10^{-4} \mathrm{~Pa}$, and the samples are exposed to air after $\mathrm{Al}$ evaporation [42, 43].

Multi-walled CNTs are grown on the devices by CVD method in a cold wall chamber (Black Magic, Aixtron SE, Herzogenrath, Germany) according 175 to the schematic process shown in Figure 2, The substrates are loaded in the chamber, which is then evacuated to a base pressure of $6.0 \mathrm{~Pa}$ with a rotary 
pump. Ammonia $\left(\mathrm{NH}_{3}\right)$ is introduced at a rate of $100 \mathrm{sccm}$ in the chamber to raise the pressure to $16 \mathrm{~Pa}$ and stabilized for $30 \mathrm{~s} . \mathrm{NH}_{3}$ is chosen as a reducing gas since it is more efficient than $\mathrm{H}_{2}$ to reduce catalyst particles to its metallic state especially at low temperature [44]. The substrate is subsequently heated up from room temperature to the growth temperature (ranging from $450{ }^{\circ} \mathrm{C}$ to $650{ }^{\circ} \mathrm{C}$ ) at a rate of $3{ }^{\circ} \mathrm{C} \cdot \mathrm{s}^{-1}$ and stabilized for $300 \mathrm{~s}$. This ramping rate is chosen as a compromise to prevent delamination of the layers while avoiding Oswald ripening of the catalyst. Upon heating, the Fe catalyst is reduced and dewets to form nanoislands and by varying the thickness of $\mathrm{Fe}$ (from $0.5 \mathrm{~nm}$ to $4.5 \mathrm{~nm}$ ), different sizes of nanoislands are formed leading to CNTs with different densities and thicknesses. After the annealing step, the carbon feedstock, acetylene $\left(\mathrm{C}_{2} \mathrm{H}_{2}\right)$, is added to commence growth. The ratio of $\mathrm{NH}_{3}$ and $\mathrm{C}_{2} \mathrm{H}_{2}$ is $1: 1$ to prevent the deposition of amorphous carbon during the growth, which is carried out at a pressure of $32 \mathrm{~Pa}$. After the growth time has elapsed, the heater is turned off, $\mathrm{C}_{2} \mathrm{H}_{2}$ and $\mathrm{NH}_{3}$ are switched off before flowing Ar $(200 \mathrm{sccm})$ until the system cooled down to room temperature as shown in Figure 2. Attempts were also made with $\mathrm{ZnO}$ as a piezoelectric material, however the $\mathrm{ZnO}$ film reacted with $\mathrm{NH}_{3}$ and is reduced to $\mathrm{Zn}$. A passivation layer that withstand the annealing step is therefore necessary to protect $\mathrm{ZnO}$ for CNT growth on the active area.

The CNTs are characterized using field emission scanning electron microscopy (FE-SEM) (LEO 1530VP scanning electron microscope, LEO electron microscopy Inc, New York, USA) using an acceleration voltage ranging from $0.5 \mathrm{keV}$ to 5 $\mathrm{keV}$. Cross-sections of cleaved samples, onto which the CNT forests have been grown, are analyzed to assess the height and forest density of the CNT layer in more detail. Area densities of the CNT forests are estimated by determining the average number of tubes across five sets of regions with $2 \mu \mathrm{m}$ distances from the SEM cross-sectional images and projecting to an approximate number of tubes over a $1 \mathrm{~cm}^{2}$ area. The CNT chemical structures are assessed by Raman spectroscopy (inVia confocal Raman microscope, Renishaw plc, Gloucestershire, UK) using a $532 \mathrm{~nm}$ wavelength laser. The scan range is chosen to be from 1000 
$\mathrm{cm}^{-1}$ to $3500 \mathrm{~cm}^{-1}$ as there were no peaks at lower wavenumbers to suggest the presence of single wall CNTs.

The electro-acoustic responses of the SMRs are characterized on a coplanar probe station using ground-signal-ground (G-S-G) probes with $150 \mu \mathrm{m}$ pitch (Picoprobes, GGB industries Inc., Naples, FL, USA). A network analyzer (Model E5062A, Keysight Technologies, Santa Rosa, CA, USA) is used to measure the frequency response of the devices from 0.5 to $3.0 \mathrm{GHz}$. The frequency corresponding to the maximum of the real part of the electrical admittance, $Y$, is considered as $f_{\mathrm{r}}$ and the frequency corresponding to the maximum of the real part of the electrical impedance, $Z$ is considered as $f_{\mathrm{a}}$. To assess the resonator performance the $Q$ value at resonance and effective electromechanical coupling, $k_{\text {eff }}^{2}$, coefficient are determined from standard definitions given by equations (1) 220 and (2) [45].

$$
\begin{aligned}
Q & =\frac{f_{\mathrm{r}}}{2}\left|\frac{\mathrm{d} \Phi_{Y}}{\mathrm{~d} f}\right|_{f=f_{\mathrm{r}}} \\
k_{\text {eff }}^{2} & =\frac{\pi}{2} \frac{f_{\mathrm{r}}}{f_{\mathrm{a}}} \frac{1}{\tan \left(\frac{\pi}{2} \frac{f_{\mathrm{r}}}{f_{\mathrm{a}}}\right)}
\end{aligned}
$$

where $\Phi_{Y}$ is the phase of $Y$.

In-liquid $Q$-factors and $Y$ spectra of both CNT coated and non-CNT SMRs are measured by carefully dropping $50 \mu \mathrm{L}$ of de-ionized (DI) water using a micropipette on top of the active area. To investigate the mass sensing ability of the SMRs, bovine serum albumin (BSA) is chosen due to its water solubility and high affinity to CNTs, which enable its adsorption on the forest. BSA solution in water with a concentration of $20 \mathrm{mg} \cdot \mathrm{mL}^{-1}(\mathrm{~B} 86657-5 \mathrm{~mL})$ is purchased from Sigma Aldrich Ltd, which is then diluted with DI water to produce three different concentrations of BSA solutions: $50 \mu \mathrm{g} \cdot \mathrm{mL}^{-1}$ (1:399 BSA:DI water), 1000 $\mu \mathrm{g} \cdot \mathrm{mL}^{-1}$ (1:19 BSA:DI water) and $2500 \mu \mathrm{g} / \mathrm{mL}$ (1:7 BSA:DI water). Pure DI water is initially dropped each device tested to obtain the reference value of $f_{\mathrm{r}}$ for no BSA binding. The DI water around the device is then carefully adsorbed using clean wipes, before allowing the device to dry for 5 mins. $50 \mu \mathrm{L}$ of one of the three concentrations of BSA solution is dropped by means of a micropipette 
235 on the SMRs covering the G-S-G probe as well and the $Y$ spectra are recorded at 2 min intervals to allow the BSA to bind to the CNT. Typically the shifts in $f_{\mathrm{r}}$ caused by BSA attachment stabilized after 5 mins. The responses to DI water and subsequently BSA for the remaining concentrations are then repeated on an SMR with CNT that has not been exposed to BSA.

\section{Results and discussion}

\subsection{CNT characterization with different catalyst thickness}

SEM images shown in Figure 3 (a) (i) and (ii) illustrate the different forest heights and densities obtained with Fe catalyst thicknesses from $0.5 \mathrm{~nm}$ to 4.5 $\mathrm{nm}$, as it is known that catalyst thickness significantly influences CNT morphology [46]. In Figure 3 (a) (i), inclined images of the forest on top of the electrode are shown, whereas in Figure 3 (a) (ii), the cross-sectional cut of the forests grown on Si wafers are depicted. From the SEM images, it is observed that when the thickness of the Fe catalyst is decreased from $4.5 \mathrm{~nm}$ to $0.5 \mathrm{~nm}$ the forest height increases from $<1 \mu \mathrm{m}$ to approximately $\sim 15 \mu \mathrm{m}$ for the same growth time of 3 minutes. In addition, a thinner catalyst layer causes the CNT forests to become more packed; hence their area density increases, as smaller nanoparticles (NPs) are formed and the individual tubes are closer to one another leading to tall VA-CNTs [47]. In contrast with Fe thicknesses of $4.5 \mathrm{~nm}$, the CNT layer tends to be more "spaghetti"-like with random orientations and

255 a lower area density, which are not ideal for biosensing. The optimum temperature to activate the Fe catalyst for the growth and to reduce delamination of the SMR reflector layers is found to be at $600{ }^{\circ} \mathrm{C}$; lower temperatures either caused slower growth rate or no growth. Higher temperatures, which could improve the CNT quality, are detrimental to the integrity of the devices as the different thermal expansion coefficients of the materials in the SMR stack lead to stress and eventually delamination. Similarly a compromise heating rate of rise of 3 ${ }^{\circ} \mathrm{C} \cdot \mathrm{s}^{-1}$ is chosen to allow NP formation and avoid film delamination during the annealing step in Stage 1. At $600{ }^{\circ} \mathrm{C}$, the growth rates are found to be approx- 
imately $5 \mu \mathrm{m} \cdot \mathrm{min}^{-1}, 3 \mu \mathrm{m} \cdot \mathrm{min}^{-1}$ and $0.2 \mu \mathrm{m} \cdot \mathrm{min}^{-1}$ for Fe thicknesses of 0.5 $\mathrm{nm}, 1.5 \mathrm{~nm}$ and $4.5 \mathrm{~nm}$ respectively as shown in Figure 3 (a) (ii). From the cross-sectional SEM micrographs, estimates for the area densities of the CNT forests are obtained as $\sim 0.3 \times 10^{10} \mathrm{~cm}^{-2}$ denoted as the low density (LD) CNTs with $4.5 \mathrm{~nm} \mathrm{Fe}, \sim 1.4 \times 10^{10} \mathrm{~cm}^{-2}$ corresponding to medium density (MD) CNTs with $1.5 \mathrm{~nm} \mathrm{Fe}$, and $\sim 2.2 \times 10^{10} \mathrm{~cm}^{-2}$ for high density (HD) CNTs with 0.5 nm Fe. Optical microscope (OM) images shown in Figure 3 (a) (iii) confirm the presence of the CNT forests on the active area of the SMRs. Shorter CNT forests are in focus with the resonator surface as in the case with $4.5 \mathrm{~nm} \mathrm{Fe}$, whereas taller CNTs are out of the focal plane in the case of $0.5 \mathrm{~nm} \mathrm{Fe}$ and 1.5 $\mathrm{nm}$ Fe. Additionally a clear change in the transparency of the active area of the 275 SMRs can be observed from the dense CNT forests to the lowest density CNT layer, indicating the reduced CNT packing with thicker Fe layers. The Raman spectra displayed in Figure 3 (b) show the presence of both a $G$-peak at 1583 $\mathrm{cm}^{-1}$ and a $D$-peak at $1350 \mathrm{~cm}^{-1}$, which are typical for multi-wall CNTs in the different CNT morphologies investigated as reported in [43]. The intensity ratio

280 of the $G$-peak to the $D$-peak $\left(I_{\mathrm{G}} / I_{\mathrm{D}}\right)$ of 1.1 with $0.5 \mathrm{~nm}$ Fe can be explained by a slightly reduced disorder in the CNT forest [48]. With $1.5 \mathrm{~nm}$ Fe the intensity of the $D$-peak becomes higher than its corresponding value for the $G$-peak indicating the deterioration of crystallinity of the CNT. Likewise with $4.5 \mathrm{~nm} \mathrm{Fe}$ the ratio $I_{\mathrm{G}} / I_{\mathrm{D}}$ is further reduced to 0.84 , hence confirming lower crystallinity in the CNTs grown with a thicker Fe layer.

\subsection{Electro-acoustic characterization of SMRs in air and DI water}

The measured $Y(S)$ spectra of the SMRs fabricated with and without CNTs are shown in Figure 4 (a). Both a TSM $(\sim 1.1 \mathrm{GHz})$ and a TLM $(\sim 1.9 \mathrm{GHz})$ resonance are observed in the spectra with a spurious resonance of smaller am290 plitude at $\sim 1.6 \mathrm{GHz}$ (Figure 4 (a)). This spurious resonance is caused by larger thicknesses in the reflector layers to compensate for temperature coefficients of frequency [49], which lead to overtones in the frequency spectra. Inclinations of the AlN grains caused by the off-axis deposition $\left(\sim 24^{\circ}\right.$, shown in Figure 1 (c)) 
lead to these quasi-shear and quasi-longitudinal resonances. These TSM and TLM resonances of the SMRs (average of ten representative devices, and standard deviation as errors) are obtained at frequencies of $(1.13 \pm 0.05) \mathrm{GHz}$ and $(1.94 \pm 0.07) \mathrm{GHz}$ respectively as shown in Figure 4 (a). Comparatively, devices with CNT forests on the active area demonstrate lower resonant frequencies of $(1.18 \pm 0.09) \mathrm{GHz}$ and $(1.91 \pm 0.06) \mathrm{GHz}$ due to the presence of the added catalyst and CNT layers. Furthermore these layers cause a reduction in $Q$ (in the range from $10 \%$ to $30 \%$ ) compared to SMRs without the CNT layer as observed in Figure 4 (b) and (c). The average $Q_{\text {air }}$ of the TSM and TLM (Figure 4 (b) and (c)) in all the devices with CNTs are smaller compared to SMRs without CNTs, due to acoustic losses added by the presence of this layer; the low density CNTs are particularly lossy because of their reduced vertical alignment as observed by SEM (Figure 3 (a)), which causes more friction-contact points. The effective electromechanical coupling coefficients, $k_{\text {eff }}^{2}$, for the TSM and TLM are found to be $(1.7 \pm 0.2) \%$ and $(3.1 \pm 0.3) \%$ respectively. Although these values are significantly inferior than those reported for AlN resonators [50, 51], it is necessary to emphasize that the focus of this work has been to achieve high $Q$, which improves the precision of determining $f_{\mathrm{r}}$ caused by low mass loads with sharper resonances.

In Figures 5 (a) and (b), the real part of the $Y(S)$ spectra of the SMRs measured in DI water with different CNT forest densities are compared with conventional SMRs without CNTs. SMRs with HD CNTs exhibit TSM and TLM resonances with strong amplitudes in DI water as compared with devices without CNTs. This is confirmed by the spectra shown in Figure 5 (a) and (b), which are normalized to their individual resonant frequencies for a clear comparison of the peak sharpness. It can be observed that the $Q$ in water of resonators with HD CNTs is 141 and 167 for the TSM and TLM respectively. Although the TSM of an SMR without CNTs has a $Q$ in water of $\sim 102$ as expected with the lower acoustic dissipation in-liquid of the shear mode, its TLM resonance is non-existent $(Q$ in water $\sim 3)$ as demonstrated by the wide resonance in Figure 5 (b). The MD CNTs and LD CNTs also have improved 
${ }_{325} Q$ in water for the TLM compared to devices without the CNTs as illustrated in Figure 5 (c). The result is significant in that the TLM has a measurable $Q$ ( $>100)$ in DI water because of the presence of the CNT layer on top. This is the first demonstration of the use of an interface layer such as a CNT forest for TLM resonance with high $Q$-factors in a liquid environment. In Figures 5 (c) 330 and (d), boxplots of the $Q$ in water of five representative devices measured are displayed for the TSM and TLM respectively. It is observed that the presence of HD CNTs on the active area isolates the resonator from the DI water leading to $Q$ larger (both for the TLM and TSM) than in the cases of the SMRs without CNTs. For the TLM $Q$ boxplot displayed in Figure 5 (d), the $Q$ in DI water increases from $\sim 3$ to $\sim 150$ as the CNT forest becomes denser, confirming the reduced contact of the DI water with the resonator surface.

\subsection{Dissipation of acoustic wave in DI water}

To quantitatively evaluate the acoustic leakage into the DI water, the dissipation factor, $D$, which is a measure of the contribution to total energy losses to the liquid medium by the resonator, is calculated using equation 3 [52].

$$
D=\frac{1}{Q_{\text {water }}}-\frac{1}{Q_{\text {air }}}
$$

where $Q_{\text {air }}$ is the unloaded $Q$ in air, which includes all losses of the resonator and $Q_{\text {water }}$ is the $Q$ in a liquid environment, which additionally includes the losses to the liquid.

In Table 1, $D$ is calculated for the TSM and it is observed that in the presence of MD and HD CNTs on the active area, $D$ improves from $(0.75 \pm 0.26) \times 10^{-2}$ to $(0.63 \pm 0.20) \times 10^{-2}$ and $(0.39 \pm 0.23) \times 10^{-2}$ respectively. Nonetheless with LD CNTs a higher acoustic leakage is observed as $D$ increases to $(1.33 \pm 0.65) \times$ $10^{-2}$, which can be caused by the smaller initial TSM $Q_{\text {air }}$ of those devices. In Table 2 the corresponding values of $D$ are calculated for the TLM and a remarkable reduction in acoustic leakage in DI water is observed ( $D$ decreases from $(31 \pm 12) \times 10^{-2}$ without CNTs to less than $2.0 \times 10^{-2}$ with the presence of the CNTs investigated). Indeed without the CNTs, the TLM suffers from large 
acoustic damping in DI water, having $Q$ values of only $\sim 3-5$, whereas with LD CNTs this $Q$ value increases to $\sim 64$, and with HD CNTs, an average $Q$ value of $\sim 160$, and $D \sim 0.45 \times 10^{-2}$ is achieved in DI water. Using taller HD CNT forests of length $30 \mu \mathrm{m}$, devices operating in a TLM with a $Q$ above 200 in water have been measured indicating that the TLM resonance is significantly isolated from the DI water droplet. This can be explained by the acoustic impedance mismatch provided by the high forest density CNT layer, which separates the resonator and the DI water. This mismatch causes the TLM to remain partially confined in the device, hence achieving a high $Q$ despite the presence of a DI water droplet.

Table 1: Quantitative analysis of the effect of different carbon nanotube (CNT) forest densities (low density (LD), medium density (MD) and high density (HD)) on the mean shear mode quality-factor in air, $Q_{\text {air }}$, quality-factor in water, $Q_{\text {water }}$, and the dissipation factor $D$ values. The standard deviation from the mean value is used to estimate the errors and variability in the values.

\begin{tabular}{lrrr}
\hline Active area & $Q_{\text {air }}$ & $Q_{\text {water }}$ & $D\left(\times 10^{-2}\right)$ \\
\hline No CNT & $320 \pm 90$ & $94 \pm 20$ & $0.75 \pm 0.26$ \\
Low density CNT & $190 \pm 60$ & $54 \pm 20$ & $1.33 \pm 0.65$ \\
Medium density CNT & $230 \pm 60$ & $94 \pm 10$ & $0.63 \pm 0.20$ \\
High density CNT & $210 \pm 40$ & $110 \pm 60$ & $0.39 \pm 0.23$ \\
\hline
\end{tabular}

Table 2: Quantitative analysis of the effect of different carbon nanotube (CNT) forest densities (low density (LD), medium density (MD) and high density (HD)) on the mean longitudinal mode quality-factor in air, $Q_{\text {air }}$, quality-factor in water, $Q_{\text {water }}$, and the dissipation factor $D$ values. The standard deviation from the mean value is used to estimate the errors and variability in the values.

\begin{tabular}{lrrr}
\hline Active area & $Q_{\text {air }}$ & $Q_{\text {water }}$ & $D\left(\times 10^{-2}\right)$ \\
\hline No CNT & $790 \pm 180$ & $3 \pm 1$ & $30.9 \pm 11.7$ \\
Low density CNT & $220 \pm 120$ & $64 \pm 40$ & $1.09 \pm 0.93$ \\
Medium density CNT & $340 \pm 110$ & $68 \pm 30$ & $1.19 \pm 0.63$ \\
High density CNT & $590 \pm 120$ & $160 \pm 60$ & $0.45 \pm 0.26$ \\
\hline
\end{tabular}




\subsection{Sensitivity to mass binding with BSA in DI water}

The effect of exposing BSA solutions of concentrations ranging from 0.05 having $\sim 15 \mu \mathrm{m}$ tall CNT layers are shown in Figure [6. A smaller (approximately by a factor of 7) relative frequency shift $\left(\Delta f_{\mathrm{r}} / f_{\mathrm{r}} \sim-39.9 \mathrm{ppm}\right)$ is demonstrated by the TSM compared to the TLM $\left(\Delta f_{\mathrm{r}} / f_{\mathrm{r}} \sim-337 \mathrm{ppm}\right)$ for the highest BSA concentration $\left(2.5 \mathrm{mg} \cdot \mathrm{mL}^{-1}\right)$, suggesting the potential for imhigher than $1 \mathrm{mg} \cdot \mathrm{mL}^{-1}$, the relative frequency shift saturates as shown in Figure 6 because the majority of the exposed binding areas on the CNT forest are occupied by BSA and no further BSA molecules can attach.

A comparison of mass binding over a time span of 8 mins for HD CNT forests 375 $Q$-factors exceeding 200 in water (inset of Figure 7 (a)), the $\Delta f_{\mathrm{r}}$ observed are negligible $(<-10) \mathrm{ppm})$ for the $30 \mu \mathrm{m}$ tall CNT forest in spite of using high BSA concentrations $\left(2.5 \mathrm{mg} \cdot \mathrm{mL}^{-1}\right)$. Indeed 8 mins after dropping the BSA on the SMR, the $\Delta f_{\mathrm{r}} / f_{\mathrm{r}}$ of the TLM is approximately $-340 \mathrm{ppm}$ with a CNT forest of $15 \mu \mathrm{m}$ in height compared to $\Delta f_{\mathrm{r}} / f_{\mathrm{r}}$ of $+6.6 \mathrm{ppm}$ for the $30 \mu \mathrm{m}$ tall CNT forest. This positive shift can be attributed to noise in the measurement or a temperature drift caused by the cooling of the resonator as the liquid evaporates. Similarly smaller changes in $f_{\mathrm{r}}(-6.2 \mathrm{ppm}$ compared to $-39.9 \mathrm{ppm})$ with the TSM modes are recorded as the CNT forest height increased from $15 \mu \mathrm{m}$ to 30 $385 \mu \mathrm{m}$. The noise in the measurement is influenced mostly by the device intrinsic response whereas the binding speed of the BSA to the CNTs affects the response time. These results suggest that the mechanical vibration to sense mass loading is dependent on the height of the CNT forest. When the CNT forest is very tall (for example $30 \mu \mathrm{m}$ ), the binding of BSA molecules on the forest does not

For biosensor applications, the TLM resonance should be able to propagate to some extent to the region where biomolecules attach -the upper part of the CNT forest is the binding region for these SMRs as shown schematically in 
Figure 7 (b). Evidently the CNT forest height needs to be tuned to have only 395 a partial isolation from the DI water while acting as a probe for biomolecule binding. Accordingly the design of the CNT forest thickness requires a compromise between acoustic isolation from the liquid and sensor mass sensitivity. The precision of this thickness tuning is however, not as critical as in the case of microchannels. Nevertheless it is necessary to identify the caveats in fabricating CNT forests to isolate the TLM resonance in liquid for biological detection. First the forest can be so tall that there is no interaction of the acoustic wave with the liquid-CNT interface as illustrated in Figure 7 (b), and second is the fact that the CNT forests easily bundle when used in liquids that have low surface tension such as isopropyl alcohol (IPA) and acetone. This type of densification consequently leads to voids or can expose the resonator surface to the liquid, causing a significant energy dissipation. In Figure 8 , the impact on the active area after immersing in different liquids and drying is shown. DI water causes no major structural damage and the CNT forest maintains its integrity as illustrated in Figure 8 (b). In this work the CNT coated SMRs have been in fact observed to retain their high $Q$-factor in DI water even after a few in-liquid measurement and dry cycles. This is a highly desirable property for applications in biosensing as the SMRs with CNTs are robust and can be re-used. In contrast there is significant exposure of a dried active area to liquids after IPA immersion shown in Figure 8 (c) and (d).

CNT coated SMRs that have been exposed to IPA have over $80 \%$ decrease in TLM $Q$-factor when used afterwards in DI water, which is close to typical TLM SMRs without CNTs, therefore confirming the higher acoustic energy dissipation into the liquid through the exposed regions. A further shortcoming of growing CNTs on gravimetric sensors is the high temperature of CVD, which renders them unsuitable for plastic substrates. As an alternative, CNTs could be grown by CVD on an independent substrate and prepared into a liquid solution using an ultrasonic spinner. CNT layers on gravimetric sensors can then be deposited either by drop-casting or spin-coating, thereby eliminating the need for high temperature processing. In this case however, dense and VA-CNTs 
425 would not be feasible and other methods such as indium-assisted transfer of VA-CNTs reported by Barako et al. [53] would be necessary.

\section{Conclusions}

In this work, the growth of CNTs of different morphologies on the active area of SMRs is achieved by CVD at $600{ }^{\circ} \mathrm{C}$ by varying the catalyst (Fe) thickness.

${ }_{430}$ The purpose of the CNT layer is to isolate the resonator active areas from liquids such as DI water to reduce acoustic damping by confining the acoustic wave in the SMR. AlN SMRs with both a TSM $(1.1 \mathrm{GHz})$ and a TLM $(1.9 \mathrm{GHz})$ resonance are fabricated to assess and compare the performance of CNT-coated devices to SMRs without CNTs in DI water. It is demonstrated that SMRs with highly packed CNT forests achieve the lowest TLM dissipation factors, $D$, of $(0.45 \pm 0.26) \times 10^{-2}$ compared to $(31 \pm 12) \times 10^{-2}$ in uncoated SMRs. Additionally TSM resonances also benefit from the CNT layer and have a lower acoustic wave leakage (a decrease in $D$ from $(0.75 \pm 0.26) \times 10^{-2}$ to $(0.39 \pm 0.23) \times 10^{-2}$ is observed) into the DI water droplet due to the presence of a highly packed CNT

${ }_{440}$ forest on the active area. Despite the presence of a tall CNT forest of up to $\sim 15$ $\mu \mathrm{m}$ height, the SMRs are sensitive to mass loading caused by BSA solutions. The result in this work is significant in that the TLM can be made to operate in DI water with the presence of a CNT isolation layer, which can potentially lead to the successful scaling up and commercialization of FBAR based biosensors.

\section{$445 \quad$ Funding}

This work was supported by the European Community's Horizon 2020 Programme [grant number SPIRE-01-2014-636820 (RECOBA)]; and the Ministerio de Economía y Competitividad del Gobierno de España [grant number MAT2013-45957-R]. G.R. and S.Z. also wish to acknowledge funding from the 450 Cambridge Commonwealth, European and International Trust. 


\section{References}

[1] G. Sauerbrey, Verwendung von schwingquarzen zur wägung dünner schichten und zur mikrowägung, Zeitschrift für Physik 155 (2) (1959) 206. doi:10.1007/BF01337937.

[2] R. Weigel, D. P. Morgan, J. M. Owens, A. Ballato, K. M. Lakin, K.-Y. Hashimoto, C. C. W. Ruppel, Microwave acoustic materials, devices, and applications, Microwave Theory and Techniques, IEEE Transactions on 50 (3) (2002) 738-749. doi:10.1109/22.989958.

[3] I. Voiculescu, A. N. Nordin, Acoustic wave based MEMS devices for biosensing applications, Biosensors and Bioelectronics 33 (1) (2012) 1-9. doi:10.1016/j.bios.2011.12.041.

[4] S. Rey-Mermet, R. Lanz, P. Muralt, Bulk acoustic wave resonator operating at $8 \mathrm{GHz}$ for gravimetric sensing of organic films, Sensors and Actuators B: Chemical 114 (2) (2006) 681. doi:10.1016/j.snb.2005.04.047.

[5] R. Aigner, SAW and BAW technologies for RF filter applications: A review of the relative strengths and weaknesses, in: Ultrasonics Symposium, 2008. IUS 2008. IEEE, 2008, pp. 582-589. doi:10.1109/ULTSYM.2008.0140.

[6] F. L. Dickert, P. Forth, W.-E. Bulst, G. Fischerauer, U. Knauer, SAW devices-sensitivity enhancement in going from $80 \mathrm{MHz}$ to 1 $\mathrm{GHz}$, Sensors and Actuators B: Chemical 46 (2) (1998) 120-125. doi:10.1016/s0925-4005(98)00097-5

[7] C.-S. Lu, O. Lewis, Investigation of film-thickness determination by oscillating quartz resonators with large mass load, Journal of Applied Physics 43 (11) (1972) 4385. doi:10.1063/1.1660931.

[8] E. Benes, Improved quartz crystal microbalance technique, Journal of Applied Physics 56 (3) (1984) 608. doi:10.1063/1.333990. 
[9] A. Flewitt, J. Luo, Y. Fu, L. Garcia-Gancedo, X. Du, J. Lu, X. Zhao, E. Iborra, M. Ramos, W. Milne, ZnO based SAW and FBAR devices for bio-sensing applications, Journal of Non-Newtonian Fluid Mechanics 222

${ }_{480}$ (2015) 209-216. doi:10.1016/j.jnnfm.2014.12.002.

[10] J. F. Rosenbaum, Bulk acoustic wave theory and devices, Artech House, New York, 1945.

[11] B. A. Auld, Acoustic fields and waves in solids, Vol. 2, Krieger Pub Co, 1990.

${ }_{485}$ [12] G. Rughoobur, M. DeMiguel-Ramos, T. Mirea, M. Clement, J. Olivares, B. Díaz-Durán, J. Sangrador, I. Miele, W. I. Milne, E. Iborra, A. J. Flewitt, Room temperature sputtering of inclined $c$-axis $\mathrm{ZnO}$ for shear mode solidly mounted resonators, Applied Physics Letters 108 (3) (2016) 034103. doi:10.1063/1.4940683.

490 [13] G. Wingqvist, AlN-based sputter-deposited shear mode thin film bulk acoustic resonator (FBAR) for biosensor applications - a review, Surface and Coatings Technology $205 \quad$ (5) (2010) 1279. doi:10.1016/j.surfcoat.2010.08.109.

[14] J. Weber, W. M. Albers, J. Tuppurainen, M. Link, R. Gabl, W. Wersing, M. Schreiter, Shear mode FBARs as highly sensitive liquid biosensors, Sensors and Actuators A: Physical 128 (1) (2006) 84-88. doi:10.1016/j.sna.2006.01.005.

[15] R. Gabl, H. D. Feucht, H. Zeininger, G. Eckstein, M. Schreiter, R. Primig, D. Pitzer, W. Wersing, First results on label-free detection of DNA and protein molecules using a novel integrated sensor technology based on gravimetric detection principles, Biosensors and Bioelectronics 19 (6) (2004) 615. doi : 10.1016/S0956-5663(03)00259-8

[16] M. Nirschl, A. Blüher, C. Erler, B. Katzschner, I. Vikholm-Lundin, S. Auer, J. Vörös, W. Pompe, M. Schreiter, M. Mertig, Film bulk acoustic resonators 
for DNA and protein detection and investigation of in vitro bacterial Slayer formation, Sensors and Actuators A: Physical 156 (1) (2009) 180-184. doi:10.1016/j.sna.2009.02.021.

[17] D. Chen, J. Wang, Y. Xu, D. Li, L. Zhang, Z. Li, Highly sensitive detection of organophosphorus pesticides by acetylcholinesterase-coated thin film bulk acoustic resonator mass-loading sensor, Biosensors and Bioelectronics 41 (2013) 163-167. doi:10.1016/j.bios.2012.08.018.

[18] G. Wingqvist, J. Bjurström, A. Hellgren, I. Katardjiev, Immunosensor utilizing a shear mode thin film bulk acoustic sensor, Sensors and Actuators B: Chemical 127 (1) (2007) 248. doi:10.1016/j.snb.2007.07.051

[19] M. DeMiguel-Ramos, T. Mirea, M. Clement, J. Olivares, J. Sangrador, E. Iborra, Optimized tilted $c$-axis AlN films for improved operation of shear mode resonators, Thin Solid films 590 (2015) 219-223. doi:10.1016/j.tsf.2015.08.010.

[20] M. DeMiguel-Ramos, B. Díaz-Durán, J.-M. Escolano, M. Barba, T. Mirea, J. Olivares, M. Clement, E. Iborra, Gravimetric biosensor based on a $1.3 \mathrm{GHz}$ AlN shear-mode solidly mounted resonator, Sensors and Actuators B: Chemical 239 (2017) (2017) 1282-1288. doi:10.1016/j.snb.2016.09.079.

[21] M. Clement, E. Iborra, J. Olivares, M. DeMiguel-Ramos, T. Mirea, ${ }_{25}$ J. Sangrador, On the effectiveness of lateral excitation of shear modes in AlN layered resonators, Ultrasonics 54 (6) (2014) 1504-1508. doi:10.1016/j.ultras.2014.04.014

[22] L. Qin, Q. Chen, H. Cheng, Q. Chen, J.-F. Li, Q.-M. Wang, Viscosity sensor using $\mathrm{ZnO}$ and AlN thin film bulk acoustic resonators with tilted polar c-axis orientations, Journal of Applied Physics 110 (9) (2011) 094511. doi:10.1063/1.3657781. 
[23] T. Yanagitani, M. Kiuchi, M. Matsukawa, Y. Watanabe, Characteristics of pure-shear mode BAW resonators consisting of (112̄0) textured $\mathrm{ZnO}$ films, Ultrasonics, Ferroelectrics, and Frequency Control, IEEE Transactions on 54 (8) (2007) 1680-1686. doi:10.1109/TUFFC.2007.439.

[24] T. Yanagitani, N. Morisato, S. Takayanagi, M. Matsukawa, Y. Watanabe, $C$-axis zig-zag $\mathrm{ZnO}$ film ultrasonic transducers for designing longitudinal and shear wave resonant frequencies and modes, IEEE Transactions on Ultrasonics, Ferroelectrics, and Frequency Control 58 (5) (2011) 1062-1068. doi:10.1109/TUFFC.2011.1906.

[25] G. Rughoobur, M. DeMiguel-Ramos, J.-M. Escolano, E. Iborra, A. J. Flewitt, Gravimetric sensors operating at $1.1 \mathrm{GHz}$ based on inclined $c$-axis ZnO grown on textured Al electrodes, Scientific Reports 7 (2017) 1367. doi:10.1038/s41598-017-01545-2.

[26] W. Xu, X. Zhang, S. Choi, J. Chae, A high-quality-factor film bulk acoustic resonator in liquid for biosensing applications, Journal of Microelectromechanical Systems 20 (1) (2011) 213-220. doi:10.1109/JMEMS.2010.2093568,

[27] W. Xu, X. Zhang, H. Yu, A. Abbaspour-Tamijani, J. Chae, In-liquid quality factor improvement for film bulk acoustic resonators by integration of microfluidic channels, Electron Device Letters, IEEE 30 (6) (2009) 647-649. doi:10.1109/LED.2009.2019973

[28] H. Zhang, E. S. Kim, Micromachined acoustic resonant mass sensor, Journal of Microelectromechanical Systems 14 (4) (2005) 699-706. doi:10.1109/JMEMS.2005.845405.

[29] S. S. Pottigari, J. W. Kwon, Vacuum-gapped film bulk acoustic resonator for low-loss mass sensing in liquid, in: Proceedings of Actuators and Microsystems Conference TRANSDUCERS 2009 - 2009 Int. Solid-State Sensors, 2009, pp. 156-159. doi:10.1109/SENSOR.2009.5285541. 
[30] M. M. J. Treacy, T. W. Ebbesen, J. M. Gibson, Exceptionally high Young's modulus observed for individual carbon nanotubes, Nature 381 (6584) (1996) 678-680. doi:10.1038/381678a0.

[31] E. Iborra, J. Sangrador, M. Clement, T. Mirea, M. DeMiguel-Ramos, J. Olivares, J. Capilla, L. García-Gancedo, S. Esconjauregui, A. J. Flewitt, W. I. Milne, Acoustic properties of carbon nanotube electrodes in BAW resonators, in: European Frequency and Time Forum International Frequency Control Symposium (EFTF/IFC), 2013 Joint, 2013, pp. 984-987. doi:10.1109/EFTF-IFC.2013.6702205.

[32] S. Wang, E. S. Humphreys, S.-Y. Chung, D. F. Delduco, S. R. Lustig, H. Wang, K. N. Parker, N. W. Rizzo, S. Subramoney, Y.-M. Chiang, A. Jagota, Peptides with selective affinity for carbon nanotubes, Nature Materials 2 (3) (2003) 196-200. doi:10.1038/nmat833.

[33] L. García-Gancedo, F. Al-Naimi, A. J. Flewitt, W. I. Milne, G. M. Ashley, J. K. Luo, X. Zhao, J. R. Lu, ZnO-based FBAR resonators with carbon nanotube electrodes, IEEE Transactions on Ultrasonics, Ferroelectrics and Frequency Control 58 (11) (2011) 2438-2445. doi:10.1109/TUFFC.2011.2100

[34] L. García-Gancedo, Z. Zhu, E. Iborra, M. Clement, J. Olivares, A. J. Flewitt, W. I. Milne, G. M. Ashley, J. K. Luo, X. B. Zhao, J. R. $\mathrm{Lu}$, AlN-based BAW resonators with CNT electrodes for gravimetric biosensing, Sensors and Actuators B: Chemical 160 (1) (2011) 1386-1393. doi:10.1016/j.snb.2011.09.083.

[35] G. Zhong, J. H. Warner, M. Fouquet, A. W. Robertson, B. Chen, J. Robertson, Growth of ultra high density single-walled carbon nanotube forests by improved catalyst design, ACS Nano 6 (4) (2012) 2893-2903. doi: $10.1021 / \mathrm{nn} 203035 \mathrm{x}$.

[36] D. N. Futaba, K. Hata, T. Yamada, T. Hiraoka, Y. Hayamizu, Y. Kakudate, O. Tanaike, H. Hatori, M. Yumura, S. Iijima, Shape-engineerable and 
highly densely packed single-walled carbon nanotubes and their application as super-capacitor electrodes, Nature Materials 5 (12) (2006) 987-994. doi:10.1038/nmat1782.

[37] H. Sugime, S. Esconjauregui, L. D’Arsié, J. Yang, T. Makaryan, J. Robertson, Growth kinetics and growth mechanism of ultra-high mass density carbon nanotube forests on conductive Ti/Cu supports, ACS Applied Materials \& Interfaces 6 (17) (2014) 15440-15447. doi:10.1021/am504048h.

[38] M. Mann, Y. Zhang, K. Teo, T. Wells, M. El Gomati, W. Milne, Controlling the growth of carbon nanotubes for electronic devices, Microelectronic Engineering 87 (5-8) (2010) 1491-1493. doi:10.1016/j.mee.2009.11.072.

[39] B. Joddar, E. Garcia, A. Casas, C. M. Stewart, Development of functionalized multi-walled carbon-nanotube-based alginate hydrogels for enabling biomimetic technologies, Scientific Reports 6 (2016) 32456. doi:10.1038/srep32456.

[40] L. García-Gancedo, E. Iborra, M. Clement, J. Olivares, Z. Zhu, A. J. Flewitt, W. I. Milne, G. M. Ashley, J. K. Luo, X. B. Zhao, J. R. Lu, Solidly mounted resonators with carbon nanotube electrodes for biosensing applications, in: Frequency Control and The European Frequency and Time Forum (FCS), 2011 Joint Conference of The IEEE International, 2011, pp. 1-5. doi:10.1109/FCS.2011.5977728.

[41] S. Esconjauregui, T. Makaryan, T. Mirea, M. DeMiguel-Ramos, J. Olivares, Y. Guo, H. Sugime, L. D’Arsié, J. Yang, S. Bhardwaj, C. Cepek, J. Robertson, E. Iborra, Carbon nanotube forests as top electrode in electroacoustic resonators, Applied Physics Letters 107 (13) (2015) 133106. doi:10.1063/1.4932197.

[42] T. Mirea, J. Olivares, M. Clement, M. DeMiguel-Ramos, J. de Frutos, J. Sangrador, E. Iborra, Carbon nanotube growth on piezoelectric AlN films: influence of catalyst underlayers, RSC Advances 5 (98) (2015) 8068280687. doi:10.1039/C5RA16840F 
[43] J. Olivares, T. Mirea, B. Díaz-Durán, M. Clement, M. DeMiguelRamos, J. Sangrador, J. de Frutos, E. Iborra, Growth of carbon nanotube forests on metallic thin films, Carbon 90 (2015) 9-15. doi:10.1016/j.carbon.2015.03.058

[44] S. Hofmann, R. Blume, C. T. Wirth, M. Cantoro, R. Sharma, C. Ducati, M. Hävecker, S. Zafeiratos, P. Schnoerch, A. Oestereich, et al., State of transition metal catalysts during carbon nanotube growth, The Journal of Physical Chemistry C 113 (5) (2009) 1648-1656. doi:10.1021/jp808560p.

[45] K. M. Lakin, Acoustic bulk wave composite resonators, Applied Physics Letters 38 (3) (1981) 125. doi:10.1063/1.92298.

[46] R. D. Bennett, A. J. Hart, R. E. Cohen, Controlling the morphology of carbon nanotube films by varying the areal density of catalyst nanoclusters using block-copolymer micellar thin films, Advanced Materials 18 (17) (2006) 2274-2279. doi:10.1002/adma.200600975

[47] J. Robertson, G. Zhong, C. S. Esconjauregui, B. C. Bayer, C. Zhang, M. Fouquet, S. Hofmann, Applications of carbon nanotubes grown by chemical vapor deposition, Japanese Journal of Applied Physics 51 (1) (2012) 01AH01. doi:10.1143/JJAP.51.01AH01.

[48] A. C. Ferrari, Raman spectroscopy of graphene and graphite: disorder, electron-phonon coupling, doping and nonadiabatic effects, Solid State Communications 143 (1-2) (2007) 47-57. doi:10.1016/j.ssc.2007.03.052.

[49] M. DeMiguel-Ramos, J. Olivares, T. Mirea, M. Clement, E. Iborra, G. Rughoobur, L. Garcia-Gancedo, A. J. Flewitt, W. I. Milne, The influence of acoustic reflectors on the temperature coefficient of frequency of solidly mounted resonators, in: 2014 IEEE International Ultrasonics Symposium, 2014, pp. 1472-1475. doi:10.1109/ULTSYM.2014.0364.

[50] M. DeMiguel-Ramos, M. Clement, J. Olivares, J. Capilla, J. Sangrador, E. Iborra, Induced surface roughness to promote the growth of tilted-AlN 
films for shear mode resonators, in: European Frequency and Time Forum International Frequency Control Symposium (EFTF/IFC), 2013 Joint, IEEE, 2013. doi:10.1109/EFTF-IFC.2013.6702076.

[51] T. Yokoyama, Y. Iwazaki, T. Nishihara, M. Ueda, Analysis on electromechanical coupling coefficients in AlN-based bulk acoustic wave resonators based on first-principle calculations, in: 2012 IEEE International Ultrasonics Symposium, Institute of Electrical and electronics Engineers (IEEE), 2012. doi:10.1109/ULTSYM.2012.0137.

[52] G. Wingqvist, J. Bjurström, L. Liljeholm, V. Yantchev, I. Katardjiev, Shear mode AlN thin film electro-acoustic resonant sensor operation in viscous media, Sensors and Actuators B: Chemical 123 (1) (2007) 466-473. doi:10.1016/j.snb.2006.09.028.

[53] M. T. Barako, Y. Gao, Y. Won, A. M. Marconnet, M. Asheghi, K. E. Goodson, Reactive metal bonding of carbon nanotube arrays for thermal interface applications, IEEE Transactions on Components, Packaging and Manufacturing Technology 4 (12) (2014) 1906-1913. doi:10.1109/TCPMT.2014.2369371.

\section{Figure legends}

Figure 1: Structure of solidly mounted resonators (SMRs) fabricated with carbon nanotubes (CNTs). (a) Schematic of the SMR structure fabricated on Si showing the 5 layer acoustic reflector, the piezoelectric AlN layer and the top electrode on which vertically aligned (VA) CNTs are grown to cover the active area. (b) A magnified view of the active area fully isolated by the presence of a forest of CNT. (c) SEM cross-section of an inclined c-axis AlN SMR demonstrating the presence of the 5 layer reflector, an Ir bottom electrode of $\sim 130 \mathrm{~nm}$ and an AlN piezoelectric film $\sim 1.2 \mu \mathrm{m}$ thick inclined at $\sim 24^{\circ}$ to the surface normal. 
Figure 2: Growth schematic with substrate heated until $600{ }^{\circ} \mathrm{C}$ at $200{ }^{\circ} \mathrm{C} / \mathrm{min}$ in $\mathrm{NH}_{3}$ (Stage 1) and stabilized to form nanoparticles (NPs) (Stage 2) $\mathrm{C}_{2} \mathrm{H}_{2}$ is added to grow carbon nanotubes (CNTs) and both $\mathrm{NH}_{3}$ and $\mathrm{C}_{2} \mathrm{H}_{2}$ are switched off (Stage 3) before cooling in Ar.

Figure 3: Characterization of carbon nanotubes (CNTs) (a) (i) Electron micrographs of CNTs grown on devices with the $0.5 \mathrm{~nm}$ Fe giving tall and high density forests. (ii) Cross-sections illustrating the heights of the CNTs and their alignment, $4.5 \mathrm{~nm}$ Fe gives randomly aligned CNTs. (iii) Optical images of the devices showing a significant contrast of the CNTs grown with $0.5 \mathrm{~nm} \mathrm{Fe}$ and $4.5 \mathrm{~nm}$ Fe as the height and density decrease. (b) Raman spectra of the CNT morphologies grown and $G$ and $D$-peaks normalized intensity ratio $\left(I_{\mathrm{G}} / I_{\mathrm{D}}\right)$, which decreases as Fe increases.

Figure 4: Electrical characterization of devices in air with and without CNTs. (a) shows the $Y(S)$ spectra of typical devices with (blue solid line) and without high density (HD) CNTs (red dashed line) on the active area, exhibiting a TSM at $f_{\mathrm{r}}$ of $1.12 \mathrm{GHz}$, a spurious mode at $1.6 \mathrm{GHz}$ and a TLM at $1.89 \mathrm{GHz}$. Graphs have been displaced for clarity. (b) Box and whisker diagram of the $Q$ in air for both the TSM at $\sim 1.1 \mathrm{GHz}$ and the TLM at $\sim 1.9 \mathrm{GHz}$ of 5 representative devices measured in each type of CNT (low density (LD), medium density (MD) and HD) showing a decrease in $Q_{\text {air }}$ due to parasitics added by CNTs. The red middle line is the median, whereas the upper and lower bounds of the box are the first and third quartiles respectively; the terminals are outliers.

Figure 5: Electrical characterization of devices in DI water. (a) Real part of the $Y(S)$ for the TSM in water for devices without CNTs, with low density CNTs (LD CNTs), medium density CNTs (MD CNTs) and high density CNTs (HD CNTs), (b) Real part of TLM $Y(S)$ in water showing resonance $\left(Q_{\text {water }}\right.$ as high as 167$)$ for all the CNT types investigated compared to SMRs without any CNTs. (c) Box and whisker diagram for the quality-factor in DI water, $Q_{\text {water }}$, for both the TSM and the TLM resonances of 5 representative devices measured in each type of SMRs illustrating the increase in $Q_{\text {water }}$ by the isolation caused by the CNT layer. The red middle line is the median, whereas the upper and lower bounds of the box are the first and third quartiles respectively; the terminals are outliers.

Figure 6: Relative resonant frequency shifts $\left(\Delta f_{\mathrm{r}} / f_{\mathrm{r}}\right)$ for the shear and longitudinal modes with different bovine serum albumin (BSA) concentrations in $\mathrm{mg} / \mathrm{mL}$. Dashed lines are guides for the eye. The longitudinal mode (blue line) with resonance frequency, $f_{1, \mathrm{r}}=1.91 \mathrm{GHz}$, has a larger shift compared to the shear mode (dark line) with resonant frequency $f_{\mathrm{s}, \mathrm{r}}=1.12$ GHz. A saturation point is reached at high concentrations. Inset shows a schematic of the droplet of BSA solution on top of the $15 \mu \mathrm{m}$ tall carbon nanotube (CNT) forest. The devices used in this test had quality-factors of $\sim 150$ in DI water. 
Figure 7: Mass sensitivity with different carbon nanotube (CNT) heights (a) Comparison of the relative frequency shifts with a BSA concentration of $2.5 \mathrm{mg} \cdot \mathrm{mL}^{-1}$ for the TSM and TLM with time for SMRs with CNTs of $\sim 15 \mu \mathrm{m}$ and $\sim 30 \mu \mathrm{m}$ in height. Lines are guides for the eye. The TLM with $15 \mu \mathrm{m}$ tall CNT has the highest $\Delta f_{\mathrm{r}}$ of $-337 \mathrm{ppm}$. Inset shows a comparison of the $Q$ in DI water for the TLM with different VA-CNT forest heights. (b) Schematic representation of the dissipation of the TLM with CNT forests of different heights, illustrating the larger amplitude resonance confined in the resonator with taller CNTs but no vibration on the surface. With shorter CNTs, acoustic wave dissipation occurs in liquid but the resonance is still significant for mass detection whereas without CNTs, all the energy is radiated in the liquid.

Figure 8: SEM images of the active area of the SMR with the HD CNTs grown, in (a) the CNT immediately after growth, (b) Dried after DI water droplet has been added, (c) Dried after IPA has been added showing exposed resonator surface (d) Voids left after IPA densification in some devices that allow liquids to penetrate the forest to the resonator. 